\title{
Relative heading estimation for pedestrians based on the gravity vector
}

\author{
Vincent Thio, Member, IEEE, Kjetil Bergh Ånonsen, and Jan Kenneth Bekkeng
}

\begin{abstract}
Inertial navigation of pedestrians carrying a smart device is a core component of many indoor positioning systems. While infrastructure-based solutions typically depend on an installation of dedicated hardware, inertial navigation depends only on sensors embedded in the device itself. A single solution can thus be applied to a large range of use cases. This work focuses on one of the main challenges in inertial navigation: user heading estimation. We describe a complete statistical model for heading estimation based on the IMU and magnetometer, assuming a fixed device pose on the pedestrian. Our aim is to provide a stand-alone solution, suitable for direct implementation into a larger positioning framework. The method consists of two consecutive parts. The first focuses on gravity vector estimation based on IMU data. We describe a method for obtaining independent estimates under dynamic conditions, thereby removing the quasi-static initialization phase required by conventional methods. The second part combines the gravity vector with gyro and magnetic measurements to estimate user heading. The proposed method is tested against a motion capture system, and against an alternative method based on attitude. We find that both methods produce similar results in terms of accuracy.
\end{abstract}

Index Terms-Accelerometer, Dead Reckoning, Gravity vector, Gyroscope, Heading, IMU, Inertial Navigation, Kalman Filter, Magnetometer, MEMS, PDR, Smartphones, Wearables

\section{INTRODUCTION}

$\mathbf{H}$ EADING estimation is a fundamental part of inertial navigation of pedestrians carrying a smart device - a concept called Pedestrian Dead Reckoning (PDR). PDR is primarily based on data obtained by the inertial measurement unit (IMU) embedded in the device. The IMU consists of a 3 -axis accelerometer and 3-axis gyroscope, and is sometimes complemented by a 3 -axis magnetometer. Inertial heading estimation models are often described in the form of an Attitude Heading Reference System (AHRS), where user heading is derived from an estimated device attitude (e.g., [1] [2] [3] [4]). A well-constrained device attitude allows for heading estimation techniques like extracting horizontal rotation [5] [6] and Principal Component Analysis (PCA) [7]. At the core of the AHRS is the sensor fusion process. This typically involves some form of Kalman Filter (KF) implementation, e.g., Extended Kalman Filter (EKF) [2] [8], Unscented Kalman Filter (UKF) [9] or Multiplicative Extended Kalman Filter

This work was supported by Forkbeard Technologies AS and the Research Council of Norway under project number 269614.

V. Thio is with the Department of Physics, University of Oslo, 0371 Oslo, Norway (e-mail: vmthio@matnat.uio.no).

K. B. Anonsen is with the Norwegian Defence Research Establishment (FFI), 2027 Kjeller, Norway, and Department of Technology Systems, University of Oslo, 0371 Oslo, Norway (e-mail: k.b.anonsen@its.uio.no

J. K. Bekkeng is with the Norwegian Defence Research Establishment (FFI), 2027 Kjeller, Norway, and Department of Physics, University of Oslo, 0371 Oslo, Norway (e-mail: j.k.bekkeng@fys.uio.no
(MEKF) [6] [10]. Other methods include a Particle Filter (PF) [3] [11] or Complimentary Filter (CF) [12].

The attitude estimation process is characterized by three distinct phases: Initialization of the device attitude, propagation based on gyro data, and periodical corrections. The latter is required to correct for accumulated errors due to residual gyro bias. Attitude propagation is a well-studied problem (e.g., [10]) and varies little between studies. Discrepancies arise in the initialization and correction phases as both depend on external measurements. A common approach is to use static periods, e.g., floor contact of a foot-mounted sensor, to obtain Zero Velocity Updates (ZUPT) [13] [14] [15]. Variations on ZUPT have been proposed to include quasi-static states of handheld devices [16] [17]. A (quasi-)static sensor allows for (re)-calibration of the gyro and direct estimation of the gravity vector. In addition, the magnetometer is used to obtain the direction, i.e., the magnetic vector, of the local magnetic field. Under ideal conditions, this vector is constantly pointing towards the magnetic North. In practice, however, indoor areas often contain local magnetic disturbances caused by electronic devices and metal in building constructions. Different techniques have been proposed to detect local disturbances and filter out reliable measurements (e.g., [18], [19]). Device attitude requires a combination of the magnetic and gravity vector to be properly defined in the local North-East-Down (NED) frame. While its accuracy thus depends on the accuracy of both these vectors, their exact estimation methods, including statistical information, are generally poorly defined. Furthermore, most 
studies require a (quasi-)static state for filter initialization and corrections (e.g., [2], [20]).

This work adds on to previous studies by giving a complete statistical derivation for independent gravity and magnetic vector estimation from raw sensor data. Our methods are valid under both static and dynamic conditions, thereby removing the (quasi-)static constraint of conventional methods. Following [6], we separate the heading estimation problem into two consecutive parts: 1) estimating the gravity vector, followed by 2) extracting the horizontal component of the gyro rate. This implicitly assumes that the change in device yaw is equal to the change in user heading. This is generally true for fixed device positions (e.g., smartwatch attached to wrist, or phone in pocket). Our work differs from [6] in that we set the gravity vector itself as Kalman filter state. Furthermore, we give an explicit description of the filter initialization phase and periodical corrections under dynamic conditions. Finally, we use the statistical information of the magnetic vector to detect local magnetic sources and filter out unreliable estimates. The methods described here are tested against the method described in [6] and against ground truth measurements based on a motion capture system. The overall aim of this study is to describe a standalone model suitable for direct implementation into a larger positioning framework.

The rest of the paper is structured as follows: Section III describes the derivation of the IMU-based gravity vector estimation method. The estimated gravity vector is used in Section IV to compute relative user heading with corrections based on the magnetometer. Experiments and results are given in Section V. Conclusions and final remarks are given in Section VI.

\section{NOTATIONS AND DEFINITIONS}

\section{A. Reference frames}

This study distinguishes between three frames of reference. Raw IMU/magnetometer measurements and gravity/magnetic unit vectors are given in the sensor frame. A special case of the sensor frame is the target frame, which is a sensor frame at zero rotation. Thus, rotation given in a sensor frame describes its absolute orientation with respect to a particular target frame. The target frame is used in Section III to rotate a windowed signal towards a common frame at window midpoint. User heading and horizontal rotation are given in the local $N E D$ frame, which is a Euclidean space described by local North, East and Down vectors. The curvature of the Earth is not considered due to the relatively small scale of typical indoor positioning problems. The focus of this study is on relative heading estimation, i.e., estimating heading relative to some initial value. In the absence of absolute heading information at initialization, this value is set to zero. Once linked to the NED frame (e.g., by PCA of the accelerometer [7]) relative heading becomes an absolute heading. To keep equations concise and readable, frame labelling is omitted whenever possible. The particular frame of reference should be clear from the context.

\section{B. Error contributions}

Errors are introduced into the system by imperfect sensor measurements. The main sources contributing to the measurement error are physical limitations of the sensor, sensor misalignment, hard/soft ironing effects and apparent drift. Errors related to physical limitations are typically described in terms of noise and bias. Sensor alignment refers to the orthogonality of the 3-axes of a single sensor, as well as the relative pose of the individual sensor frames. Sensors are aligned if they share a single common frame with orthogonal axes. In addition, magnetic measurements are distorted by magnetic components (hard ironing) and temporal magnetic variations due to current fluctuations (soft ironing) of the device containing the sensor [21]. Errors caused by a fixed bias, misalignment and hard/soft ironing effects can be reduced by proper sensor calibration. We assume calibrated sensors throughout this work. Residual gyro bias is of particular interest in inertial navigation as it causes attitude drift over time. While negligible in gravity vector estimation (Section III), it does affect the heading estimation process described in Section IV. Temporal variations of gyro bias are beyond the scope of this paper. Instead, filtered magnetometer measurements are used for periodical heading corrections.

An additional gyro error source is Earth's rotation, causing apparent drift of the attitude. The gyro noise of commercialof-the-shelf smart devices is generally larger than the magnitude of the Earth's rotation. Apparent drift effects are thus absorbed by the sensor noise and not explicitly modelled.

We assume additive white Gaussian noise for all sensors, defined by zero mean and standard deviation obtained by experiment. The sensor noise implicitly contains residual calibration errors, temporal changes in sensor bias and apparent drift. Finally, we assume uncorrelated noise between sensors.

\section{Notation conventions}

Matrices are described by upper case, rotation matrices by $R$ and covariance matrices by $P$ (e.g., $P_{f}$ ). The variance of a scalar (e.g., heading $\psi$ ), is described by a 1-by-1 'variance matrix' $P_{\psi}$ for consistency. Vectors are given in bold, where the distinction is made between a true value $f$, expected value $\bar{f}$ (sometimes referred to as an estimated or predicted value) and corrected value $\hat{f}$. Raw measurements, or values based directly thereupon, are described by a tilde, $\tilde{f}$. The $s$ superscript indicates a smoothed value, and the $r$-superscript a vector representation in the target frame. $k$-subscripts indicate timesteps in discrete systems. Error increments $\delta \boldsymbol{f}$ are either described by a normal distribution, e.g., $\boldsymbol{\delta} \boldsymbol{f} \sim \mathcal{N}\left(\boldsymbol{\mu}_{f}, \boldsymbol{\sigma}_{f}\right)$, or in terms of 'lower-level' errors. The 3-by-3 skew-symmetric matrix of a vector $\boldsymbol{f}$ is given by $[\boldsymbol{f}]_{\times}$. Specifics regarding the quaternion notation are given in the relevant section.

\section{ill. Gravity vector estimation}

The accelerometer measures the specific force, denoted $f$, over its three axes of acceleration. The specific force is the sum of the instantaneous user acceleration $\boldsymbol{a}$ and the local gravity $\boldsymbol{g}$ with an opposite sign, formalized as

$$
\boldsymbol{f}(t)=\boldsymbol{a}(t)-\boldsymbol{g}(t)
$$


where all vectors are represented in the sensor frame. In the following derivation, the time dependency $t$ will be dropped. Separating the local gravity into a magnitude scalar times a unit directional vector gives

$$
\boldsymbol{g}=g \cdot \gamma
$$

Where $g$ is the local gravity magnitude, and $\gamma$ is the local gravity unit vector to be estimated. Under static conditions, the user acceleration is zero and the specific force is directly related to the local gravity by

$$
\boldsymbol{f}=-\boldsymbol{g} \quad \text { and } \quad\|\boldsymbol{f}\|=g,
$$

which gives the unit gravity vector as

$$
\gamma(\boldsymbol{f})=-\frac{\boldsymbol{f}}{\|\boldsymbol{f}\|} .
$$

The specific force as measured by the accelerometer is subject to noise. The measured value, denoted $\tilde{f}$, is thus the true specific force minus an unknown error $\delta \boldsymbol{f}$,

$$
\tilde{f}=f-\delta f .
$$

The error is described in terms of the diagonal matrix $\tilde{P}_{f}$, defined by the vector $\sigma_{f}$ containing the three uncorrelated standard deviations of the accelerometer. In a similar way, the true gravity vector is the sum of its 'measured' value (i.e., directly related to $\tilde{f}$ ), denoted $\tilde{\gamma}$, plus an unknown error $\delta \gamma$. The expected value follows directly from (4) by setting $f=\tilde{f}$. The relationship between $\delta \boldsymbol{\gamma}$ and $\delta \boldsymbol{f}$ can be approximated by a first order Taylor expansion,

$$
\begin{aligned}
\gamma & =\tilde{\gamma}+\boldsymbol{\delta} \gamma \\
& \approx \tilde{\gamma}+H_{\tilde{f}} \delta f
\end{aligned}
$$

where $H_{\tilde{f}}$ is the Jacobian of (4), evaluated at $\tilde{\boldsymbol{f}}$, given by

$$
H=\frac{\partial \boldsymbol{\gamma}(\boldsymbol{f})}{\partial \boldsymbol{f}}=\frac{1}{\|\boldsymbol{f}\|}\left(I_{3}-\frac{1}{\|\boldsymbol{f}\|^{2}} \boldsymbol{f} \boldsymbol{f}^{T}\right) .
$$

The covariance matrix of the 'measured' gravity vector can now be obtained as follows,

$$
\tilde{P}_{\gamma}=H_{\tilde{\boldsymbol{f}}} \tilde{P}_{\boldsymbol{f}} H_{\tilde{\boldsymbol{f}}}^{T}
$$

The general process of IMU-based gravity vector estimation thus comes down to approximating (4) and (8) by removing user acceleration and noise from the acceleration signal. For a pedestrian carrying a phone, the embedded IMU is in a dynamic state due to the walking motion. This translates into a periodic IMU signal, approximately repeated every gait cycle (a gait cycle consists of two steps, i.e., comparable posture at start and end). The IMU frequency is thus bounded below by the walking frequency, except during transition periods (i.e., start of walk), when true acceleration/deceleration occurs. Note, however, that lower frequencies might still occur due to slight changes in walking velocity and sensor movements unrelated to the walking motion.

\section{A. Low-pass filtering of the acceleration signal}

A first approach to the gravity vector estimation problem is thus to apply a low-pass filter to the acceleration signal with a cut-off frequency well below the walking frequency. This approach was previously described by [6], who use an Infinite Impulse Response (IIR) filter with cut-off frequency around $0.3 \mathrm{~Hz}$. Here, a Finite Impulse Response (FIR) filter is used instead. This is to stay consistent with the extended methods described later on. Only the discrete version of the filter will be evaluated here, since measurements typically come in at discrete intervals. The discrete filter can be formalized as follows,

$$
\boldsymbol{f}_{k}^{s}=\sum_{i=-N / 2}^{N / 2} b_{i} \boldsymbol{f}_{k+i}
$$

where $b_{i}$ is the $i^{t h}$ weight of the discretized smoothing window of size $N$. The corresponding covariance $P_{\boldsymbol{f}_{k}^{s}}$ can be obtained by computing the standard deviation over the individual axes of the windowed signal.

The gravity vector derived this way diverts from its true value due to residual user acceleration, sensor noise and 'short-term' rotation of the sensor. Residual acceleration and noise effects can be reduced by increasing the window size, effectively lowering the cut-off frequency. Short-term rotation refers to the dynamics occurring within the time interval of the smoothing window. These are primarily related to the walking motion. This in contrast to 'long-term' dynamics, e.g., gradual changes in user heading. The short-term rotation effects derive from the implicit assumption that the sensor does not rotate during the smoothing process, i.e., the direction of gravity is equal for all timesteps in the window. In practice, the sensor rotates at approximately the walking frequency.

This constitutes a trade-off problem: reducing the window size reduces the dynamic effects, but also reduces the effectiveness of the low-pass filter. A way to mitigate the shortterm rotation effects is by using the rotation rate, obtained from the gyroscope, to rotate the windowed signal towards a single common frame. This 'rotation-adjusted' method will be discussed next.

\section{B. Adjusting for short-term rotation}

The orientation, or attitude, of the sensor if often described in the form of a quaternion to ensure a non-singular representation. The quaternion, denoted $\boldsymbol{q}(t)$, is defined by

$$
\boldsymbol{q}(t)=\left[q_{w}, \boldsymbol{q}_{v}^{T}\right]^{T}
$$

where $q_{w}$ is the scalar and $\boldsymbol{q}_{v}^{T}$ the vector part. The quaternion is related to the rotation rate by the kinematic equation,

$$
\frac{d}{d t} \boldsymbol{q}(t)=\frac{1}{2} \Omega(\boldsymbol{\omega}(t)) \cdot \boldsymbol{q}(t)
$$

where $\omega(t)$ is the rotation rate represented in the sensor frame and $\Omega$ is defined by

$$
\Omega(\boldsymbol{\omega}(t))=\left[\begin{array}{cc}
0 & -\boldsymbol{\omega}(t)^{T} \\
\boldsymbol{\omega}(t) & -[\boldsymbol{\omega}(t)]_{\times}
\end{array}\right] .
$$


Assuming a constant rotation rate between gyro measurements $\boldsymbol{\omega}_{k}$, the discretized closed-form solution to (11) becomes (see e.g., [6], [10]),

$$
\boldsymbol{q}_{k+1}=\Delta \boldsymbol{q}_{k} \otimes \boldsymbol{q}_{k}
$$

where $\otimes$ denotes the quaternion product and

$$
\Delta \boldsymbol{q}_{k}=\boldsymbol{q}\left(\boldsymbol{\omega}_{k} \Delta t\right)=\left[\begin{array}{c}
\cos (\alpha) \\
\boldsymbol{\beta} \sin (\alpha)
\end{array}\right]
$$

with

$$
\alpha=\left\|\boldsymbol{\omega}_{k}\right\| \Delta t / 2, \quad \boldsymbol{\beta}=\frac{\boldsymbol{\omega}_{k}}{\left\|\boldsymbol{\omega}_{k}\right\|}
$$

constitutes the rotation between $t_{k}$ and $t_{k+1}$. The rotation action is based on the rotation matrix, denoted $R$, which is related to the quaternion by

$$
R(\boldsymbol{q})=\left(q_{w}^{2}-\boldsymbol{q}_{v}^{T} \boldsymbol{q}_{v}\right) I+2 \boldsymbol{q}_{v} \boldsymbol{q}_{v}^{T}+2 q_{w}\left[\boldsymbol{q}_{v}\right]_{\times}
$$

Rotation of a vector, e.g. an acceleration measurement $\boldsymbol{f}(t)$, then follows from direct matrix-vector multiplication,

$$
\boldsymbol{f}^{r}(t)=R(\boldsymbol{q}(t)) \boldsymbol{f}(t)
$$

where $\boldsymbol{f}^{r}(t)$ is the representation of $\boldsymbol{f}(t)$ in the target (rotation free) frame. The opposite rotation, i.e., representing a 'target frame'-vector in the sensor frame, can be achieved by applying the quaternion conjugate $\boldsymbol{q}^{*}=\left[q_{w},-\boldsymbol{q}_{v}^{T}\right]^{T}$. This is equal to taking the transpose of the rotation matrix.

The 'low-pass filter' method can now be extended by applying local rotation to the windowed signal. The extended version of the discrete low-pass filter (9) becomes,

$$
\boldsymbol{f}_{k}^{r s}=\sum_{i=-N / 2}^{N / 2} b_{i} R\left(\boldsymbol{q}_{k, k+i}\right) \boldsymbol{f}_{k+i}
$$

where $\boldsymbol{q}_{k, k+i}$ denotes the rotation between $t_{k}$ and $t_{k+i}$. This rotation results from repeated application of (13),

$$
\boldsymbol{q}_{k, k+i}= \begin{cases}\prod_{j=0}^{i} \boldsymbol{q}\left(\boldsymbol{\omega}_{k+j} \Delta t\right), & \text { for } i>0 \\ \prod_{j=0}^{i} \boldsymbol{q}^{*}\left(\boldsymbol{\omega}_{k+j} \Delta t\right), & \text { for } i<0 \\ {[1,0,0,0]^{T},} & \text { for } i=0\end{cases}
$$

where $\prod$ denotes the repeated quaternion product. The covariance matrix $P_{\boldsymbol{f}_{k}^{r s}}$ follows from the standard deviations of the individual axes after adjustment for rotation.

The 'rotation-adjusted' method provides an independent gravity vector estimate at any point in time, similar to the 'lowpass filter' method but with reduced dynamic effects. Since the short-term rotation effects are actively integrated into the solution, the trade-off problem of the previous method is no longer present. While the rotation adjustment step does add an additional error source in the form of gyro noise, its effects are limited to the length of the window. For window sizes up to a couple of seconds, as used in this study, the gyro noise effects are considered negligible.

The rotation-adjusted gravity vector estimates are independent, i.e., each estimate places a full weight on its own value. The next step is to include information from previous estimates. This is particularly useful when going from a static state, yielding high-accuracy estimates, into a dynamic state. This 'fusion-based' approach is described next.

\section{Fusion based estimation}

A detailed description of an MEKF setup for IMU-driven systems is given by [10]. The basic idea of the MEKF is to compute an unconstrained estimate of the three error components, and use them to update the predicted (and unit normalized) four-component quaternion. This provides a globally non-singular attitude representation where the a posteriori updated quaternion is a unit vector within first order. A brute force normalization is typically applied after the measurement update equation. An MEKF setup focusing specifically on gravity vector estimation was previously described by [6]. The MEKF is a relatively complex way of estimating the gravity vector from IMU data. An alternative method, presented below, is to replace the MEKF by a simpler EKF and setting the gravity vector itself as filter state. In addition, gravity vector estimates based on the 'rotation-adjusted' method provide periodical state corrections. To ensure independent measurements, the time between measurements should be equal or larger than the window size $N$. The periodical corrections remove the quasi-static period constraint typically required for filter initialization. The EKF setup is described as follows:

a) initialization: The filter initializes after $N$ timesteps with an independent measurement $\tilde{\gamma}_{0}$ and associated covariance matrix $\tilde{P}_{\gamma_{0}}$ :

$$
\hat{\gamma}_{0}=\tilde{\gamma}_{0}, \quad \hat{P}_{\gamma_{0}}=\tilde{P}_{\gamma_{0}}
$$

b) propagation: Incoming gyro measurements are integrated into a rotation matrix and the gravity vector is rotated accordingly. The statistical information of the unknown gyro error, $\boldsymbol{\delta} \boldsymbol{\omega}$, cannot be added directly to $\hat{P}_{\gamma}$. Instead, the rotation matrix is separated into its expected value and an error component. Using the residual of the second term of the Taylor expansion for the error, we get

$$
\begin{aligned}
R\left(\boldsymbol{\omega}_{k} \Delta t\right) & =R\left(\left(\tilde{\boldsymbol{\omega}}_{k}+\boldsymbol{\delta} \boldsymbol{\omega}_{k}\right) \Delta t\right) \\
& \approx R\left(\tilde{\boldsymbol{\omega}}_{k} \Delta t\right)+\left[\boldsymbol{\delta} \boldsymbol{\omega}_{k} \Delta t\right]_{\times}
\end{aligned}
$$

where $R\left(\boldsymbol{\omega}_{k} \Delta t\right)=R\left(\Delta \boldsymbol{q}_{k}\right), \tilde{\boldsymbol{\omega}}$ is the measured rotation and $\delta \boldsymbol{\omega}$ the unknown error based on the gyro noise. From this, the expected value becomes

$$
\bar{\gamma}_{k+1}=R\left(\tilde{\boldsymbol{\omega}}_{k} \Delta t\right) \hat{\gamma}_{k} .
$$

The propagation of the covariance matrix is given by

$$
\bar{P}_{\gamma_{k+1}}=R\left(\tilde{\boldsymbol{\omega}}_{k} \Delta t\right) \hat{P}_{\gamma_{k}} R\left(\tilde{\boldsymbol{\omega}}_{k} \Delta t\right)^{T}+\left[\hat{\gamma}_{k}\right]_{\times} Q\left[\hat{\gamma}_{k}\right]_{\times}^{T}
$$

where the diagonal matrix $Q$ contains the uncorrelated gyro variances integrated over time (i.e., $\boldsymbol{\sigma}_{\omega}^{2} \Delta t^{2}$ ).

c) correction: The state correction, based on a gravity vector measurement $\tilde{\gamma}_{k}$ with $\tilde{P}_{\gamma_{k}}$, is a straightforward process. The standard Kalman filter equations apply with $H=I_{3}$,

$$
\begin{aligned}
K_{k} & =\bar{P}_{\gamma_{k}}\left(\bar{P}_{\gamma_{k}}+\tilde{P}_{\gamma_{k}}\right)^{-1} \\
\hat{\gamma}_{k} & =\bar{\gamma}_{k}+K_{k}\left(\tilde{\gamma}_{k}-\bar{\gamma}_{k}\right) \\
\hat{P}_{\gamma_{k}} & =\left(I_{3}-K_{k}\right) \bar{P}_{\gamma_{k}}\left(I_{3}-K_{k}\right)^{T}+K_{k} \tilde{P}_{\gamma_{k}} K_{k}^{T}
\end{aligned}
$$


where $\bar{\gamma}_{k}$ and $\bar{P}_{\gamma_{k}}$ are the a priori, and $\hat{\gamma}_{k}$ and $\hat{P}_{\gamma_{k}}$ the a posteriori state estimates. The gravity vector should be renormalized after correction due to errors introduced by the approximation step of (21) and rounding errors.

\section{HEADING ESTIMATION}

A well-constrained gravity vector allows for decomposition of any vector in the sensor frame into its NED horizontal and vertical components. Thus, given a current relative or absolute heading estimate $\psi_{k}$, the updated heading $\psi_{k+1}$ becomes

$$
\psi_{k+1}=\psi_{k}+\boldsymbol{\gamma}_{k}^{T} \boldsymbol{\omega}_{k} \Delta t
$$

where $\gamma_{k}$ is the gravity vector and $\boldsymbol{\omega}_{k}$ the rotation rate at time $t_{k}$. The expected value of $\psi_{k+1}$ follows directly from (25) by substituting $\hat{\psi}_{k}, \hat{\gamma}_{k}$ and $\tilde{\boldsymbol{\omega}}_{k}$. The heading error $\delta \psi$ relates to the errors in gravity vector and rotation rate as follows,

$$
\psi_{k+1}=\psi_{k}+\left(\hat{\gamma}_{k}+\boldsymbol{\delta} \gamma_{k}\right)^{T}\left(\tilde{\boldsymbol{\omega}}_{k}+\boldsymbol{\delta} \boldsymbol{\omega}_{k}\right) \Delta t
$$

where $\psi=\bar{\psi}+\delta \psi$. This gives the variance of $\psi_{k+1}$ as

$$
\bar{P}_{\psi_{k+1}}=\hat{P}_{\psi_{k}}+\hat{\gamma}_{k}^{T} Q \hat{\gamma}_{k}+\tilde{\boldsymbol{\omega}}_{k}^{T} \hat{P}_{\gamma_{k}} \tilde{\boldsymbol{\omega}}_{k} \Delta t^{2}
$$

where $Q$ contains the integrated gyro noise and $\hat{P}_{\gamma_{k}}$ is the covariance matrix of the gravity vector.

\section{A. Heading corrections based on the magnetometer}

The 3-axis magnetometer measures the magnetic field flux density (MFFD), denoted $B$, in the sensor frame. The direction of $B$ is given by a unit vector $\boldsymbol{\mu}$, from hereon referred to as the magnetic vector. The magnetic vector is derived from raw magnetometer measurements following a similar process as described in Section III. This gives a mean value $\overline{\boldsymbol{\mu}}_{k}$ and covariance matrix $P_{k}$ of the magnetic vector at time $t_{k}$. The magnetic vector indicates the horizontal orientation, or yaw, of the phone with respect to the NED frame. It is, however, not a direct measurement of the user heading itself. Instead, two sets of measurements, taken at $t_{1}$ and after some time at $t_{2}$, can be used to estimate the change in heading $\Delta \psi$ over that time interval. The process is summarized as follows.

The first step is to compute the vector normal to the plane described by the magnetic/gravity vector set $\left(\boldsymbol{\mu}_{i}, \boldsymbol{\gamma}_{i}\right)$, at $t_{i=1}$ and $t_{i=2}$. This vector, denoted $\boldsymbol{e}_{i}$, is given by

$$
\boldsymbol{e}_{i}=\gamma_{i} \times \boldsymbol{\mu}_{i}
$$

and lies in the horizontal plane of the NED-frame in approximate Eastward direction. Substituting $\gamma_{i}=\hat{\gamma}_{i}$ and $\boldsymbol{\mu}_{i}=\tilde{\boldsymbol{\mu}}_{i}$ yields the 'measured' value $\tilde{e}_{i}$. The corresponding covariance matrix of $\boldsymbol{e}$ is given by

$$
\tilde{P}_{\boldsymbol{e}_{i}}=\left[\hat{\gamma}_{i}\right]_{\times} \tilde{P}_{\boldsymbol{\mu}_{i}}\left[\hat{\gamma}_{i}\right]_{\times}^{T}+\left[\tilde{\boldsymbol{\mu}}_{i}\right]_{\times} \hat{P}_{\boldsymbol{\gamma}_{i}}\left[\tilde{\boldsymbol{\mu}}_{i}\right]_{\times}^{T} .
$$

Since both $\boldsymbol{e}_{1}$ and $\boldsymbol{e}_{2}$ lie in the horizontal plane, $\Delta \psi$ is equal to the angle between the two vectors,

$$
\pm \Delta \psi=\cos \left(\frac{\boldsymbol{e}_{1}^{T} \boldsymbol{e}_{2}}{\left\|\boldsymbol{e}_{1}\right\| \cdot\left\|\boldsymbol{e}_{2}\right\|}\right),
$$

where we select the solution closest to the value obtained by the estimation step (25). The 'measured' value of $\Delta \psi$ follows directly from (30) by substituting $\tilde{e}_{1}$ and $\tilde{e}_{2}$, giving $\Delta \tilde{\psi}$. The covariance of $\Delta \psi$ can be approximated using a first-order Taylor expansion,

$$
\begin{aligned}
\Delta \psi & =\Delta \tilde{\psi}+\delta \psi \\
& \approx \Delta \tilde{\psi}+H_{\tilde{\boldsymbol{e}}^{a}} \boldsymbol{\delta} \boldsymbol{e}^{a}
\end{aligned}
$$

where $e^{a}$ is an augmented vector containing both $e$-vectors and $H_{\tilde{\boldsymbol{e}}^{a}}$ is the Jacobian of $\Delta \psi$ evaluated at $\tilde{\boldsymbol{e}}^{a}$. The Jacobian can be computed by applying the chain rule,

$$
H=\frac{\partial \Delta \psi}{\partial \boldsymbol{e}^{a}}=\frac{\partial \Delta \psi}{\partial f\left(\boldsymbol{e}^{a}\right)} \frac{\partial f\left(\boldsymbol{e}^{a}\right)}{\partial \boldsymbol{e}^{a}}
$$

where

$$
f\left(e^{a}\right)=\frac{e_{1}^{T} e_{2}}{\left\|e_{1}\right\| \cdot\left\|e_{2}\right\|} .
$$

The first component of (32), a scalar, is given by the cosine derivative. The second component is a vector described by

$$
\frac{\partial f\left(\boldsymbol{e}^{a}\right)}{\partial \boldsymbol{e}^{a}}=f\left(\boldsymbol{e}^{a}\right)\left[\begin{array}{l}
\frac{\boldsymbol{e}_{1}}{\boldsymbol{e}_{1}^{T} \boldsymbol{e}_{2}}-\frac{\boldsymbol{e}_{2}}{\left\|\boldsymbol{e}_{1}\right\|} \\
\frac{\boldsymbol{e}_{2}}{\boldsymbol{e}_{1}^{T} \boldsymbol{e}_{2}}-\frac{\boldsymbol{e}_{1}}{\left\|\boldsymbol{e}_{2}\right\|}
\end{array}\right] .
$$

Substituting (32)-(34) into (31), the variance of $\Delta \tilde{\psi}$ becomes

$$
\tilde{P}_{\Delta \psi}=H_{\tilde{\boldsymbol{e}}^{a}}^{T} \tilde{P}_{e^{a}} H_{\tilde{\boldsymbol{e}}^{a}}
$$

where $\tilde{P}_{e^{a}}$ is the covariance matrix of the augmented vector $\tilde{\boldsymbol{e}}^{a}$. The magnetometer-based heading measurement at $t_{2}$ becomes

$$
\begin{gathered}
\tilde{\psi}_{2}=\tilde{\psi}_{1}+\Delta \tilde{\psi}_{1,2} \\
\tilde{P}_{\psi_{2}}=\tilde{P}_{\psi_{1}}+\tilde{P}_{\Delta \psi_{1,2}}
\end{gathered}
$$

where the 1,2-subscript indicates the dependence on gravity and magnetic vector measurements at $t_{1}$ and $t_{2}$. Finally, the heading correction is computed from the weighted average of the magnetometer-based heading measurement (36) and the IMU-based heading prediction (25)-(27), where the weights are determined by the variances.

The magnetic vector measurement model is based on an undisturbed magnetic field. That is, it is assumed that the magnetic vector points towards the same direction in the NED frame at $t_{1}$ and $t_{2}$. This condition requires a filtering procedure to ensure only measurements with relatively low magnetic disturbance are considered. We set the following two conditions:

- Condition I - a magnitude check,

$$
\operatorname{abs}(\tilde{B}-B c)>\varepsilon_{m}
$$

Where $\tilde{B}$ is the measured MFFD value, $B c$ the expected value based on an external model (e.g., World Magnetic Model) and $\varepsilon$ a tolerance parameter based on magnetometer and model errors. The measurement is rejected if it exceeds the tolerance parameter. This is the standard and straightforward method to remove clear outliers.

- Condition II - A motion/variance check. Applying the 'rotation-adjusted' method to the magnetometer yields a vector mean and variance. In the absence of local magnetic sources, the variance is small regardless of device motion. The variance is also small in the presence of a 
local magnetic source if the device is static. If, however, the device is in motion, then a local magnetic source results in a large variance. This concept is demonstrated more clearly in the third experiment of Section V.

\section{EXPERIMENTS AND RESULTS}

In this section, we evaluate the described methods by experiment. The setup consists of a ground truth reference system based on motion capture by Qualysis [22], calibration of the 'real' data source (iPhone 8), and determination of the model parameters (e.g., filter type, sensor standard deviations). In the first experiment we evaluate the gravity vector estimation process for three common phone positions. The most challenging phone position (phone in pocket) is used in the second experiment to estimate user heading. Finally, we use simulated local magnetic sources to demonstrate the effectiveness of the magnetometer filtering method.

\section{A. Data acquisition}

1) Ground Truth measurements: The MoCap system consists of 24 cameras (Miqus M3) covering an area of approximately $60 \mathrm{~m}^{2}$ at a tracking rate of $300 \mathrm{~Hz}$. The system uses optical tracking to obtain positions of individual reflectors in the local NED frame. Reflectors placed on three distinct corners of the phone facilitate rigid body tracking (Fig. 1). This gave a $97 \%$ coverage and near-continuous tracking of the 6-DoF of the phone at sub-millimeter accuracy. During postprocessing, the ground truth measurements were converted into simulated phone attitude, IMU data and user heading. Time synchronization between the MoCap system and iPhone is based on cross-correlation between simulated and real IMU data.

2) Phone measurements: Accelerometer, gyroscope and magnetometer data were gathered using an iPhone 8 at a measurement rate of $100 \mathrm{~Hz}$. A preliminary recording, made under static conditions, was used to quantify the noise levels of the individual sensors. The noise is described by a single standard deviation per sensor (i.e., independent of sensor axis) for the accelerometer $(1 \mathrm{e}-3 \mathrm{~g})$, gyroscope $(2 \mathrm{e}-3 \mathrm{rad} / \mathrm{s})$ and magnetometer $(0.2 \mu T)$.

\section{B. Experiment l: Gravity vector estimation}

The first experiment evaluates the gravity vector estimation process for three common phone positions, i.e., 'Calling', 'Navigating' and 'Pocket' (Fig. 1). A fourth scenario, 'fast pace' with phone in the pocket, was added in addition. Each scenario consists of 10 seconds of walking. The gravity vector is estimated using four different methods: 'low-pass filtered' (LPF, see Section III-A), 'rotation-adjusted' (RA, see Section III-B), 'gravity vector-based KF' (GKF, see Section III-C) and 'attitude-based KF' (AKF, taken from [6]). The window size was set to $N=200$ to approximate a low-pass filter with $\sim 0.3 \mathrm{~Hz}$ cut-off frequency, following [6]. The periodical correction interval of the KF methods is thus two seconds. The accuracy of the individual methods is quantified by computing the norm of the difference between the estimated $\left(\gamma_{e}\right)$ and ground truth $\left(\gamma_{g t}\right)$ gravity vector as follows,

$$
\epsilon=\left\|\gamma_{e}-\gamma_{g t}\right\| .
$$

where $\epsilon$ is the error norm.

The results, given in terms of $\epsilon$, are shown in Fig. 2. The 'Calling' and 'Navigating' scenarios produce similar results for all methods, and generally better than both 'Pocket' scenarios. Holding the phone in hand reduces the effect of the walking motion on the phone, which results in a relative constant gravity vector. The walking motion has a significant effect, however, on a phone in the pocket. The benefit of the RA and KF methods becomes clear in this scenario, where the walking motion is clearly visible in the LPF results. Errors increase when walking at a faster pace, which may be due to increased user acceleration.

Differences between the RA, GKF and AKF methods are small. Note, however, that the AKF method was initialized using a 'perfect' gravity vector estimate based on the MoCap system, whereas the initial value of the GKF method is based on real IMU data. The 'Pocket' scenario is used in the next experiments since it is the most challenging scenario.

\section{Experiment II: User heading}

The second experiment focuses on heading estimation based on Section IV. The experiment consists of a phone in a pocket for a walking trajectory of 40 seconds including multiple 180 degrees turns (Fig. 3). The accuracy of the estimated heading profile for each gravity vector estimation method is quantified as the difference between the estimated heading and ground truth heading. The results are shown in Fig. 4. As shown, all methods perform similarly with the exception of the LPF method.

\section{Experiment III: Filtering magnetic data}

The test area did not contain significant magnetic sources. Heading based on the magnetometer thus produced low errors $\left(<1^{\circ}\right)$ along the entire walking trajectory. To simulate the effect of local disturbances, four virtual magnetic sources were introduced into the test area, shown in grey in Fig. 3. The magnetic disturbances were added to the raw magnetometer measurements to obtain a 'disturbed' signal.

Fig. 5 shows the MFFD (top) and magnetic vector variance norm (bottom) for the undisturbed and disturbed signals. The MFFD plot shows minor differences between the signals, which means that the local sources have little effect on the magnitude of the magnetic field. Condition I (Section IV-A) would thus be insufficient to filter out local magnetic sources. The magnetic variance plot, on the other hand, clearly indicates proximity to local magnetic sources. The local sources thus have a strong effect on the local direction of the magnetic field. These local directional changes are filtered out based on Condition II. 


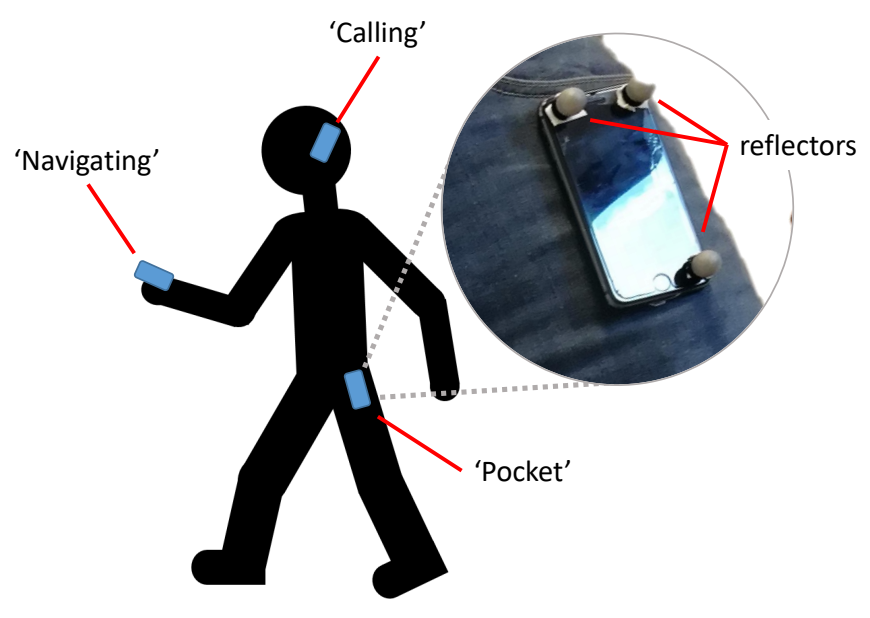

Fig. 1. The three fixed phone positions considered in this study. The 'Pocket' position is heavily influenced by short-term rotations related to the walking motion, whereas the 'Calling' and 'Navigating' positions are relatively stable. The 'Pocket' position is thus the more challenging scenario (see also Fig. 2). Rigid body tracking is based on the three reflectors in the corners of the phone.

TABLE I

GRAVITY VECTOR ERRORS MEAN AND STD

\begin{tabular}{|r||c|c|c|l|}
\cline { 2 - 5 } \multicolumn{1}{c|}{} & \multicolumn{1}{c|}{ LPF } & \multicolumn{1}{c|}{ RA } & \multicolumn{1}{|c|}{ GKF } & \multicolumn{1}{|c|}{ AKF } \\
\hline Calling & $0.08(0.05)$ & $0.06(0.03)$ & $0.04(0.02)$ & $0.04(0.02)$ \\
Navigating & $0.06(0.03)$ & $0.05(0.03)$ & $0.03(0.01)$ & $0.04(0.02)$ \\
Pocket & $0.28(0.13)$ & $0.12(0.08)$ & $0.09(0.06)$ & $0.1(0.06)$ \\
Fast pace & $0.22(0.1)$ & $0.15(0.08)$ & $0.14(0.07)$ & $0.14(0.07)$ \\
\hline
\end{tabular}

\section{Conclusions}

This study describes a complete statistical model for heading estimation based on the IMU and magnetometer. It includes a detailed description of the derivation of the gravity and magnetic vector estimates, including statistical information, from raw sensor data under dynamic conditions. The model consists of two consecutive steps, 1) gravity vector estimation, followed by 2) extraction and integration of the horizontal component of the rotation rate. The first step uses either the latest gravity vector estimate (RA method) or all estimates up to that point using an EKF setup (GKF method). Experimental results show that the accuracy of both methods are similar to those obtained by an attitude-based (AKF) method described by [6]. The RA method is of particular interest as it does not require a Kalman filter setup and is thus better suited for low-performance devices. In addition, we apply the statistical information of the magnetic vector to detect local magnetic disturbances, filter out unreliable magnetic measurements, and correct the (relative) user heading. The proposed method is validated by experiment using simulated local magnetic sources. For a pedestrian in motion, we find that the proposed method clearly detects areas with local magnetic disturbances.
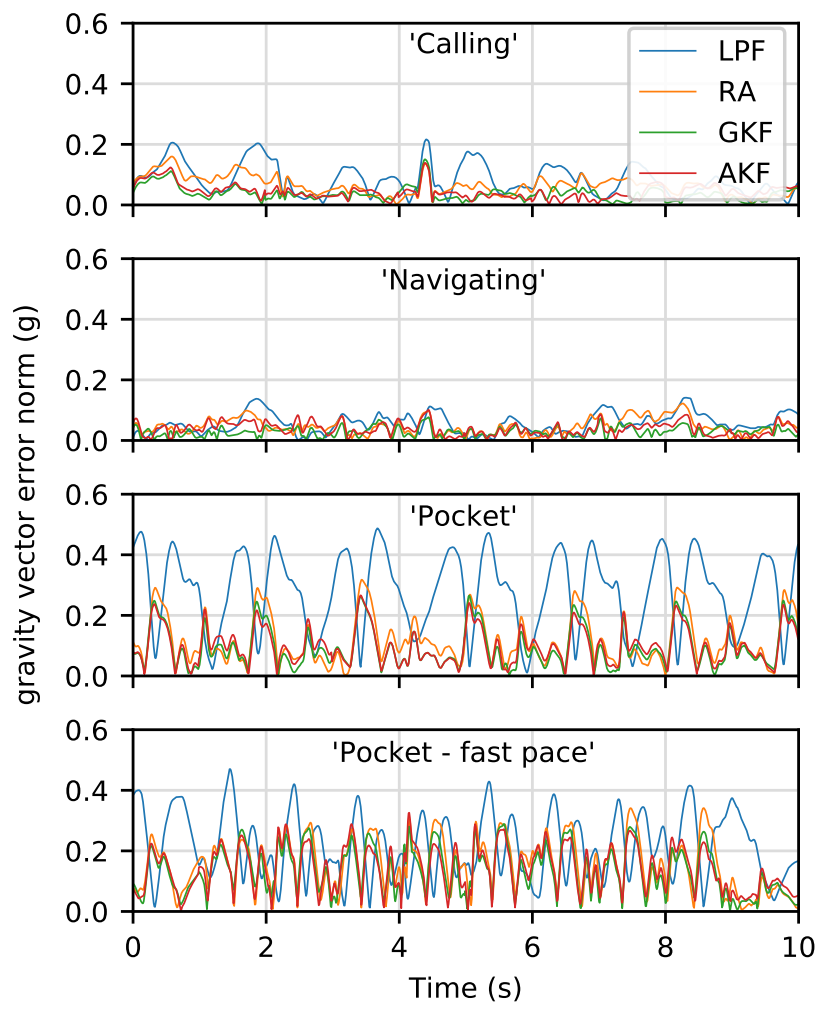

Fig. 2. Calculated gravity vector error norm for the three phone positions, plus a 'fast pace' experiment with the phone in the pocket, over a 10 second walking period. Details per method given in the relevant sections. All methods yield relatively small errors for the 'Calling' and 'Navigating' phone positions. The 'Pocket' position, which is strongly affected by the walking motion, shows that applying short-term rotation adjustment reduces the error significantly. Walking at a faster pace reduces the effect of applying short-term rotations, which may be due to increases in the user acceleration.

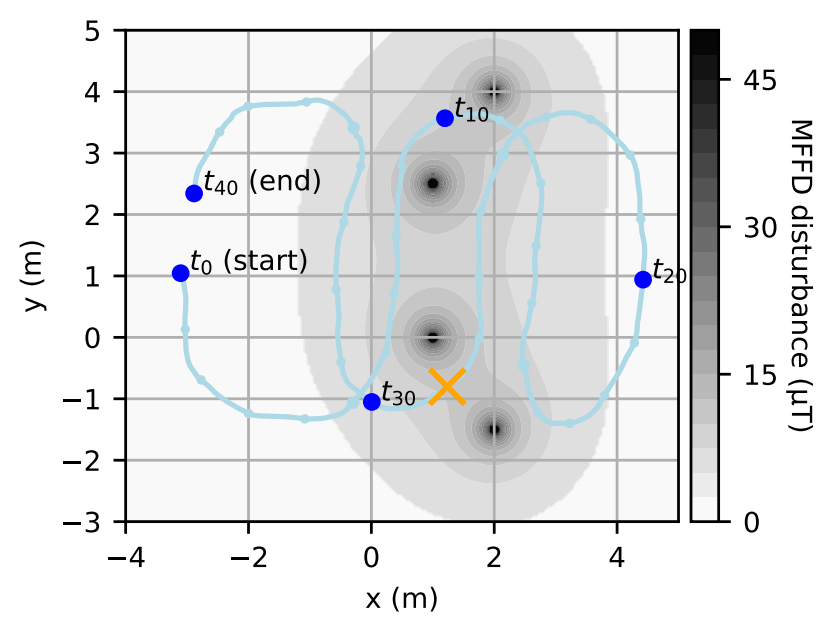

Fig. 3. 40 seconds walking trajectory as recorded by the MoCap system (blue). In black the four simulated local magnetic sources with associated MFFD field in grey scale. The orange symbol represents largest magnetic vector variance along the trajectory, see also Fig. 5. 


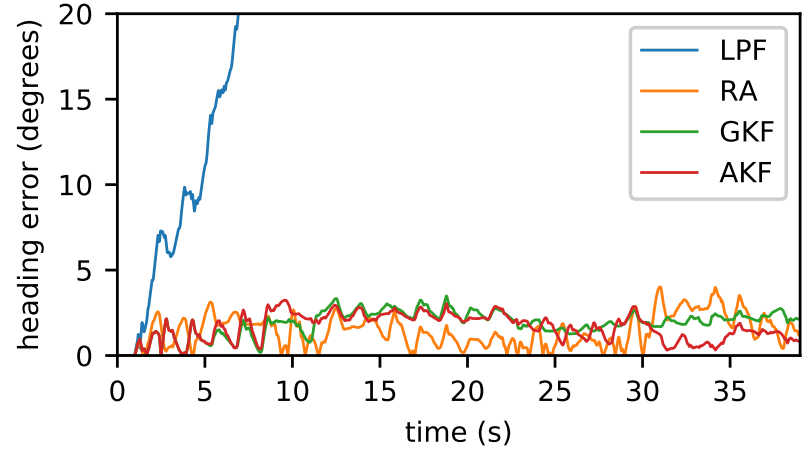

Fig. 4. Heading error computed as the absolute difference between estimated heading and ground truth heading. Ground truth heading based on simulated phone attitude and gravity vector.
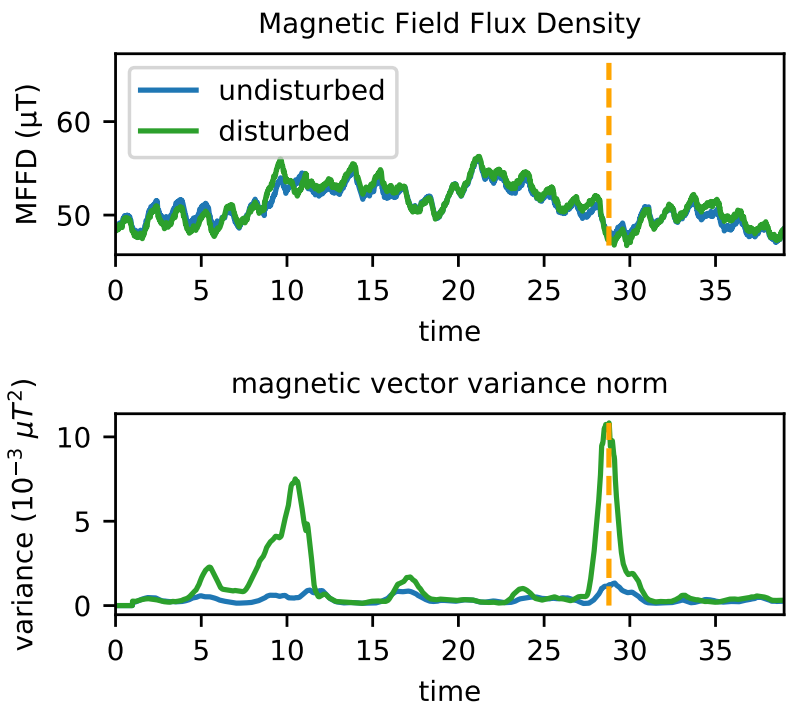

Fig. 5. Magnetic Field Flux Density (top) and computed magnetic vector variance (bottom) along the walking trajectory. 'Undisturbed' signals (blue) based on the magnetometer only, whereas 'disturbed' signals include simulated magnetic sources. Orange line indicates largest variance. The results show the benefit of using magnetic vector variance over MFFD. Local magnetic sources may change the orientation of the magnetic field without explicitly changing the overall magnitude. The variance method clearly indicates proximity to local magnetic sources at 10 and 29 seconds.

\section{REFERENCES}

[1] M. Basso, M. Galanti, G. Innocenti, and D. Miceli, "Pedestrian dead reckoning based on frequency self-synchronization and body kinematics," IEEE Sensors Journal, vol. 17, no. 2, pp. 534-545, 2016.

[2] J. Kuang, X. Niu, and X. Chen, "Robust pedestrian dead reckoning based on MEMS-IMU for smartphones," Sensors, vol. 18, no. 5, p. 1391, 2018.

[3] L. Pei, D. Liu, D. Zou, R. Lee Fook Choy, Y. Chen, and Z. He, "Optimal heading estimation based multidimensional particle filter for pedestrian indoor positioning," IEEE Access, vol. 6, pp. 49705-49720, 2018.

[4] J. Qian, L. Pei, J. Ma, R. Ying, and P. Liu, "Vector graph assisted pedestrian dead reckoning using an unconstrained smartphone," Sensors, vol. 15, no. 3, pp. 5032-5057, 2015.

[5] G. Chen, X. Meng, Y. Wang, Y. Zhang, P. Tian, and H. Yang, "Integrated $\mathrm{WiFi} / \mathrm{PDR} /$ smartphone using an unscented Kalman filter algorithm for 3D indoor localization," Sensors, vol. 15, no. 9, pp. 24595-24614, 2015.

[6] A. Manos, I. Klein, and T. Hazan, "Gravity-based methods for heading computation in pedestrian dead reckoning," Sensors, vol. 19, no. 5, p. 1170, 2019.
[7] Z.-A. Deng, G. Wang, Y. Hu, and D. Wu, "Heading estimation for indoor pedestrian navigation using a smartphone in the pocket," Sensors, vol. 15, no. 9, pp. 21518-21536, 2015.

[8] V. Renaudin and C. Combettes, "Magnetic, Acceleration Fields and Gyroscope Quaternion (MAGYQ)-Based Attitude Estimation with Smartphone Sensors for Indoor Pedestrian Navigation," Sensors, vol. 14, no. 12, pp. 22864-22890, 2014.

[9] X. Yuan, S. Yu, S. Zhang, G. Wang, and S. Liu, "Quaternion-based unscented Kalman filter for accurate indoor heading estimation using wearable multi-sensor system," Sensors, vol. 15, no. 5, pp. 10872-10890, 2015.

[10] J. Sola, "Quaternion kinematics for the error-state Kalman filter," arXiv:1711.02508, 2017.

[11] N. Yadav and C. Bleakley, "Accurate orientation estimation using AHRS under conditions of magnetic distortion," Sensors, vol. 14, no. 11, pp. 20008-20024, 2014.

[12] M. B. Del Rosario, N. H. Lovell, and S. J. Redmond, "Quaternion-based complementary filter for attitude determination of a smartphone," IEEE Sensors Journal, vol. 16, no. 15, pp. 6008-6017, 2016.

[13] E. Foxlin, "Pedestrian tracking with shoe-mounted inertial sensors," IEEE Computer graphics and applications, vol. 25, no. 6, pp. 38-46, 2005.

[14] W. T. Faulkner, R. Alwood, D. W. Taylor, J. Bohlin, and D. David, "Gpsdenied pedestrian tracking in indoor environments using an imu and magnetic compass," in Proceedings of the 2010 International Technical Meeting of the Institute of Navigation, pp. 198-204, Citeseer, 2010.

[15] M. Ma, Q. Song, Y.-h. Li, Y. Gu, and Z.-m. Zhou, "A heading error estimation approach based on improved quasi-static magnetic field detection," in 2016 International Conference on Indoor Positioning and Indoor Navigation (IPIN), pp. 1-8, IEEE, 2016.

[16] M. N. Muhammad, Z. Salcic, I. Kevin, and K. Wang, "Indoor pedestrian tracking using consumer-grade inertial sensors with pztd heading correction," IEEE Sensors Journal, vol. 18, no. 12, pp. 5164-5172, 2018.

[17] Q. Zeng, S. Zeng, J. Liu, Q. Meng, R. Chen, and H. Huang, "Smartphone heading correction based on gravity assisted and middle time simulatedzero velocity update method," Sensors, vol. 18, no. 10, p. 3349, 2018.

[18] V. Renaudin, M. H. Afzal, and G. Lachapelle, "Magnetic perturbations detection and heading estimation using magnetometers," Journal of Location Based Services, vol. 6, no. 3, pp. 161-185, 2012.

[19] B. Fan, Q. Li, and T. Liu, "How magnetic disturbance influences the attitude and heading in magnetic and inertial sensor-based orientation estimation," Sensors, vol. 18, no. 1, p. 76, 2018.

[20] Z.-A. Deng, Y. Hu, J. Yu, and Z. Na, "Extended Kalman filter for real time indoor localization by fusing WiFi and smartphone inertial sensors," Micromachines, vol. 6, no. 4, pp. 523-543, 2015.

[21] D. Liu, L. Pei, J. Qian, L. Wang, C. Liu, P. Liu, and W. Yu, "Simplified ellipsoid fitting-based magnetometer calibration for pedestrian dead reckoning," in Proc. China Satellite Navigation Conference (CSNC), (Singapore), pp. 473-486, Springer, 2016.

[22] https://www.qualisys.com/. 